\title{
In vitro 3D Model of Female Reproductive Tract: An Overview and Future Aspect
}

\author{
Arnaud Martino Capuzzo \\ Department of Veterinary Medicine, University of Milan, Italy
}

\begin{abstract}
Hormones must be balanced and dynamically controlled for the Female Reproductive Tract (FRT) to function correctly during the menstrual cycle, pregnancy, and delivery. Gamete selection and successful transfer to the uterus, where it implants and pregnancy occurs, is supported by the mucosal epithelial lining of the FRT ovaries, uterus, cervix, fallopian tubes, and vagina. Successful implantation and placentation in humans and other animals rely on complex interactions between the embryo and a receptive female reproductive system. The FRT's recent breakthroughs in three-dimensional (3D) organoid systems now provide critical experimental models that match the organ's physiological, functional, and anatomical characteristics in vitro. This article summarizes the current state of the art on organoids generated from various parts of the FRT. The current analysis examines recent developments in the creation of organoid models of reproductive organs, as well as their future directions.
\end{abstract}

Keywords: Female Reproductive Tract, Organoid, Co-culture, Crosstalk, Blastocyst.

\section{Introduction}

The female reproductive system has a unique function in that it includes the beginning of life while simultaneously having the ability to end it. The vagina, cervix, uterus, endometrium, and fallopian tubes are all composed of mesodermal derivatives, whereas the ovaries are constructed of endoderm. The development, proliferation, and differentiation patterns of each of these tissues are distinct (Mancini \& Pensabene, 2019).

Importantly, in order to fulfil their reproductive role, these tissues rely on the capacity of their constituents to self-renew. However, it is in their efforts to allow life to begin by performing transportation functions, assisting with implantation, and acting as barriers to internal and external stressors and pathogens that its constituents risk cellular deregulation as a result of 
threatening impacts such as hormonal dysregulation, infections, or auto-immune diseases, which can lead to carcinogenesis. (N. Desai et al., 2012).

In humans and other mammals, successful implantation and placentation are reliant on elaborate interactions between the embryo and a receptive female reproductive system (Fritz et al., 2014).

Early embryonic death is a common side effect of infertility. At the conclusion of the perimplantation period, up to $60 \%$ of all pregnancies are terminated. The disturbance of the embryomaternal communication is one of the main reasons for this (Herrler et al., 2003).

The time interval preceding conception and nidation (implantation) or attachment of the embryo to the uterus has been identified as the preimplantation stage of development in mammals. The preimplantation period lasts about 30, 7, 4, and 5 days in cattle, humans, mice, and rats, respectively. The zygote undergoes numerous cleavage divisions after fertilization, dividing the ooplasm into several equal-sized compartments termed blastomeres. The zona pellucida surrounds the blastomeres as a whole. (Madan, 2011).

The blastocysts are firmly enclosed by the maternal endometrium, according to ultrasound findings (Madsen et al., 2015; Meyer et al., 2019). The extra-embryonic matrix (EEM, zona pellucida) represents the interface between mother and embryo because the human embryo does not hatch until day 6 . However, signals during the first 6 days should therefore pass through this matrix to reach their destination, so EEM can be considered as a mail box when studying embryomaternal signaling (Herrler et al., 2003).

\section{In vitro 3D model of Female Reproductive Tract}

In general, various study models that have evolved through time to become increasingly complex and representative, and even personalized, have provided insight into tissue development, homeostasis, and disease. Hypothesis testing for FRT tissues has depended on two-dimensional (2D) models (such as cell lines) for many years, alongside short-term three-dimensional (3D) in vitro cell-culture and in vivo explant systems. However, it wasn't until 2009 that the idea of a total shift to long-term 3D in vitro cell-culturing technologies was envisioned (Heremans et al., 2021).

Numerous biological insights have been gained through 2D cultivation of immortalized cell lines or those generated from cancer in organs of interest (Haycock, 2011; 3D Model Systems, 2020). Their benefits include repeatability and accessibility. However, they lack many complex aspects 
of in vivo microenvironments, such as cell-cell/cell- Extracellular Matrix (ECM) interactions, and they fail to replicate important properties of native tissues, such as active cell signaling pathways and function, due to changes in cell lines (Heidari-Khoei et al., 2020).

Traditional 2D culture systems, which grow alone or in co-culture on plates and conduct experiments supported by in vitro imaging for various functional, pharmacological, toxicological, and even clinical applications, have long been widely used and are well-known for their low cost and high repeatability. Due of the limitations associated with the lack of particular tissue architecture, mechanical-biochemical signals, cell-cells, and ECM, 2D culture methods cannot achieve a degree of 3D organization comparable to in vivo. (A. M. Capuzzo et al., 2020; A. M. Capuzzo \& Vigo, 2021).

The 3D cell cultures, total display improved work, yet need cell extremity and the 3D association that is available in vivo. Other 3D culture frameworks incorporate spheroids that frequently come up short on the limit with regards to self-organization and separation because of the shortfall of applicable cell ancestor or undeveloped cells (Łaniewski et al., 2017; Yin et al., 2016) .

Organ explants and organotypic slice cultures that mimic the native organ's complex 3D architecture, cellular heterogeneity, and function are extremely useful for studying development and physiology, but they are limited by their inability to proliferate, short lifespan, and cell phenotype drift due to improper culture media orientation. Organoid technology is a novel in vitro model that may be utilized as tissue and organ proxies to bridge the gap between in vitro and in vivo research. (S. P. Desai et al., 2013).

The innovation of organoids in the last decade offers new in vitro models to be considered as intermediaries of both tissues and organs to overcome any problems between in vitro and in vivo (Lancaster \& Knoblich, 2014; Choi et al., 2016). Organoids constructed and validated over time repeat elements of various target organs. In this way, the fallopian tubes, the uterus and only the trophoblast as organoids of the ovaries were made in the research sights for the problems related to FRT. These models are opening new roads to explore the typical science and pathology of the FRT.

Organoids are miniature replicas of self-organizing structures that mimic many biological and pathological characteristics of organs, including several reproductive organs. Organoids of the 
ovaries, uterus, fallopian tubes, and as well as trophoblasts, have been generated in the laboratory in this regard. Current achievements in building organoid models of reproductive organs as well as future directions will be highlighted.

\section{Endometrial organoids}

To prepare for embryo implantation, the endometrium undergoes cyclic modifications in response to steroid hormones, as well as paracrine and autocrine factors. Embryo implantation is a complicated procedure that necessitates a receptive endometrium, a capable blastocyst, and a coordinated maternal-embryo interaction. Dysmenorrhea, infertility, endometrial polyps, endometrial cancer and endometriosis, which is the most frequent cancer of the female reproductive organs, are all associated with the endometrium (Burkitt et al., 2012).

Blauer created and confirmed a culture condition in which normal human endometrium was grown as glandular organoids in a Matrigel matrix alongside stromal cells for the first time. However, this 3D culture method diverged from the commonly used organoid concept in terms of principles and protocols. In 2017, two different groups reported successful endometrial organoids production in mice and human endometria. Dissociated endometrial cells were implanted in Matrigel droplets in culture medium that is routinely used to facilitate the growth of organoid models of other organs to create these endometrial organoids (Chumduri \& Turco, 2021)

The endometrial organoids' molecular and functional characteristics were identical to those of their parent cells. Endometrial organoids, like in vivo endometrium, feature glandular-type selforganization, apicobasal polarity, and functional activity, such as mucus production, in addition to being sex hormone sensitive (Chen \& Schoen, 2019).

Some endometrial organoids have been obtained from endometrial and adjacent endometrial adenocarcinomas from postmenopausal subjects. This starting condition has helped grow the small amount of material adopted, for an assortment of high-throughput assessments, and could be a significant platform for: examining implantation problems, histotrophic sustenance period in early pregnancy, new procedures useful for gynecological pathologies such as endometriosis and malignant endometrium growth and create an endometrial biobank (Senol et al., 2016).

In contrast to healthy endometrial-derived organoids, the tumor organoids showed a variety of patient-specific morphologies, including pleomorphic cells with hyperchromic nuclei, 
disorganized epithelium and basement membrane breakdowns, and invasion of the isolated cells to the surrounding Matrigel. The organoids generated from the tumor were however positive for the MUC1 and SOX17 glandular markers, confirming their glandular origins (Elad et al., 2020).

Research on the chromosome stability of endometrial organoids utilizing the CGH array showed that the organoids created had maintained their genetic integrity for many months in culture. These genetically stable endometrial organoids might be produced and maintained to create a patient-specific biobank of endometrial tissues without losing their proliferative potential after thawing. The culture system has the ability to expand a small number of starting materials in a variety of high-performance assessments, including implantation, the histotrophic nutritional period during pregnancy, new gynecological pathologic therapy strategies for endometriosis and endometrial cancer, and the creation of an endometrial biological bank (Xiao et al., 2017).

The contemporary organoids, on the other hand, are devoid of stromal cells. The physiological roles of endometrial epithelial and stromal cells, as well as the genesis of numerous pathologic diseases such as endometrial cancer, are well understood. Endometrial organoids will never have the context they need to be true mini organs until they have a fuller complement of cell types (Stejskalová et al., 2021).

Co-culturing endometrial organoids with stromal cells may improve the organoid model and provide a suitable paradigm for investigating reciprocal epithelium-stroma interactions in vivo. The inaccessible luminal compartment and apical appearance of the epithelium of organoids make physiologically relevant research difficult. A luminal compartment might be created by unfolding the spherical organoid into a 2D planar tissue construct and monolayer culture of primary epithelial cells from organoids (Stejskalová et al., 2021).

\section{Ovarian organoids}

The ovaries oversee oocyte maturation and release into the fallopian tubes on a cyclical basis. They also operate as endocrine glands, secreting estrogen and progesterone, which work together to orchestrate the endometrium's cyclical changes. The ovary, like other reproductive organs, is dynamic, as seen by the ovarian surface epithelium's (OSE) periodic rupture and healing (Schomberg, 1978).

There haven't been any long-term cultures of healthy ovarian epithelium described yet. To study the relationship between chronic inflammation and ovarian cancer, the first 3D cultures of OSE 
were produced(Wong \& Auersperg, 2003). Tissue-derived cultures of OSE from women with benign gynecological diseases were seeded on Matrigel-coated wells overlayed with 2 percent Matrigel in medium based on 3D cultures of mammary epithelial acini (Kwong et al., 2009).

Single cell-layered epithelial spheroids with a central lumen produced, surrounded by a basement membrane. The spheroids had apical polarity but were unable to develop over time. Continuous TNF treatment resulted in a precancerous phenotype in the spheroids, with enhanced proliferation and loss of apicobasal polarity. More recently, 3D cultures from OSE have been obtained from BRCA1/BRCA2 mutation carriers with a high risk of ovarian cancer (Alzamil et al., 2021).

Furthermore, ovarian cancer organoids have somatic mutations and amplifications/deletions, and their marker expression and mutational landscape are similar to that of the parent tumor (Maenhoudt et al., 2020). Other ovarian illnesses, such as premature ovarian failure and polycystic ovary, might be used to create organoids to research the processes that cause these diseases (Heidari-Khoei et al., 2020).

\section{Fallopian tube organoids}

Fallopian tubes are bilateral ducts that connect the uterine body to the ovaries. The fallopian tube (also known as the oviduct or uterine tube) is a vital organ in the female reproductive system that aids egg maturation and selection, gamete and embryo transit, sperm reservoir, polyspermy control, fertilization, and early embryonic development (Heidari-Khoei et al., 2020).

Heidari-Khoei in his work, exposes how two main methods for the realization of organoids of the fallopian tubes, have different problems (Heidari-Khoei et al., 2020). Because such culture methods lack tubular folded structures and do not allow for live imaging or perfusion investigations, they are only useful for studying gamete interactions and early embryo development in depth. The fallopian tube was created in self-organizing organoids using a modified intestine organoid technique. Isolated fallopian epithelial cells were planted in a 2D culture, then cultured in a 3D Matrigel matrix with growth factors (EGF, FGF, and TGF-), niche specific factors (Wnt3a, R-spondin-1 [RSPO1], ALK4/5, and Noggin), and an anoikis inhibitor (ROCK inhibitor) (Kessler et al., 2015).

The organoid configurations of the fallopian tubes described closely resemble the in vivo counterparts' normal physiology and morphology. In terms of screening technologies, cancer 
biology, and reproductive medicine, they provide potential models for investigating the biology and pathology of the fallopian tubes (Alzamil et al., 2021; Wong \& Auersperg, 2003). However, although these systems present limitations for the interaction studies between gametes or embryos due to their small size, they could be considered if they were to be used for cross-talk communication between FRT structures. The ability to recreate and validate an in vitro system considering the multitude of factors present is still far away.

\section{Trophoblast organoid}

The placenta is an additional embryonic organ that is necessary for the mammalian embryo's survival and growth. The trophectoderm layer of the blastocyst adheres to the endometrial epithelium after implantation in humans and continues to differentiate into three forms of trophoblasts: extravillous CTB (EVT), cytotrophoblast (CTB), and syncytiotrophoblast (STB) (Laronda et al., 2013).

CTB cells that are not differentiated develop through the STB to create cell columns and chorionic villi. EVT infiltrate the deciduum when CTB cells at the tips of villi develop into EVT. Endovascular EVTs enter and remodel the spiral arteries, while interstitial EVT cells invade and remodel the decidualized endometrium. Nutrient exchange and synthesis are carried by multinucleated STB cells. To keep the pregnancy going, they emit placental hormones such placental lactogen, chorionic gonadotropin, and progesterone, as well as a variety of other proteins and steroids. Interstitial CTB cells fuse together to form these cells (Li et al., 2011).

Major pregnancy-related problems such as intrauterine growth restriction, preeclampsia, miscarriage, repeated abortion, and preterm labor are caused by placental malfunction and insufficiency. Due to a paucity of relevant functional models, our understanding of the human placenta is restricted. The placenta has been studied using a variety of experimental methods, including animal models and in vitro cell culture models. Human and other animal placentas, on the other hand, have different structural and functional features, therefore data from animal models is not applicable to people (Haider et al., 2018).

Placenta-derived cell lines, isolated primary placenta cells, and human placenta tissue explants are all in vitro models of human trophoblasts. JEG-3, BeWo, and JAR are three cell lines derived from choriocarcinoma cells. Easy use, endless supply of material, purer cell source, less expensive to purchase, convenience of use for gene silencing techniques, and these sources avoid some of 
the ethical considerations involved with the use of animal or primary human tissues are all advantages of these cell lines (Turco et al., 2018).

Immortal cell lines, on the other hand, have a number of drawbacks as a result of their malignant transformations. The HTR-8/ SVneo cell line, which was created by transfecting first trimester EVT cells with the simian virus 40 large T antigen gene, or the AC1-M59 and ACH-3P cell lines, which are choriocarcinoma cells fused with primary first and third trimester trophoblast cells, respectively, can be used to solve this problem. (Turco et al., 2018).

However, some of these cells don't meet the requirements for human trophoblast cells because they lack critical protein markers (GATA3, KRT7, and TFAPC2), the HLA class I profile, the chromosome 19 miRNA cluster (C19MC), and hypomethylation of the ELF5 promoter. Alternative models have been created, such as human embryonic stem cells (hESCs) treated with bone morphogenetic protein 4 (BMP4) (Sheridan et al., 2020).

Despite the fact that these trophoblast-like cells are used as models to investigate early trophoblast lineage segregation, their global gene expression patterns, trophoblast-specific markers, and HLA status are considerably different from those of primary trophoblast populations. Organoid platforms for in vitro growth of first-trimester CTBs were recently developed by two separate research groups. Turco described seeding specific first-trimester placenta development cell clusters into Matrigel drops and culturing them in a trophoblast organoid medium (TOM) containing FGF2, EGF, A83-01, CHIR99021, and RSPO (Deloria et al., 2021).

Hepatocyte growth factor (HGF), PGE2, and Y-27632 were added to improve cell survival and growth. Within a week, the researchers found rapid cell expansion when TOM was used. When villous CTBs (vCTBs) were embedded in Matrigel with a predetermined cocktail of growth factors and signaling inhibitors, tiny cell clusters formed within a few days of culture, according to Haider et al. After 2-3 weeks, these clusters grew quickly and turned into organoids with uneven structures (Abbas et al., 2020).

Organoid structures appeared after 10-14 days in these two well-defined environments, and homogenous trophoblast organoids were generated. The prenatal origin of trophoblast organoids was confirmed by microsatellite analysis and HLA typing. The trophoblast organoids were 
genetically stable and showed mitochondrial activity following cryopreservation after more than 6 months of passages. Finally, they showed that the organoids were clonal in origin and that massive organoids could be grown from single cells in 3-4 weeks (Horii et al., 2020).

The identities of these trophoblast organoids were verified against trophoblast-specific criteria at similar or higher levels than the choriocarcinoma lines JEG-3 and JAR. Principal component analysis of placental villi, trophoblast organoids, placental stromal cells, and decidual organoids using 12,673 probes revealed that the trophoblast organoid clusters were more closely related to the placenta, with enrichment for trophoblast-specific genes such as GATA3, CGB3, and PSG6 (Sheridan et al., 2020).

The trophoblast organoid structures had a surrounding foundation membrane beneath the SCT, VCT in the middle of the organoids, numerous secretory organelles, syncytial masses that lined the central cavity, and surface microvilli that nearly mirrored the organization of placental villi in vivo. Proteomic examination of the conditioned medium by liquid chromatography-tandem mass spectrometry (LC-MS/MS) revealed the secretory function of trophoblast organoids (Li et al., 2011).

Peptides, hormones, and enzymes secreted by trophoblast organoids include pregnancy-specific glycoprotein (PSG), early placental insulin-like protein (INSL4 or EPIL), human chorionic gonadotropin (hCG), growth differentiation factor 15 (GDF15), kisspeptin (KISS1), chorionic somatomammotropin hormone 1 (CSH1), and aldose reductase. Both endovascular and interstitial EVT express HLA-G, a strong tolerogenic molecule at the maternal-fetal interface, and its expression grows as trophoblasts move towards the spiral arteries (Haider et al., 2018).

A few HLA-G+ cells were discovered in trophoblast organoids in basic organoid medium. HLAG+ cells that had migrated out of the organoids appeared in the 3D culture and invaded it, adhering to the plastic dish. As a result, the organoids resembled the villous placenta anatomically, functionally, metabolically, and endocrinologically. Overall, the trophoblast organoid model appears to be a potential tool for studying human placental development and trophoblast malfunction and insufficiency. Researchers would be able to explore the mechanisms that underpin maternal-fetal interactions during pregnancy if they developed an advanced culture system that included both endometrial and trophoblast organoids (Elad et al., 2020). 


\section{General method and tips for Organoid Culture: from planning to imaging}

To create a 3D organoid structure, is always needed to start from a source. Organoids can be cultured from donor tissue, progenitor organ cells, embryonic and pluripotent stem cells induced stem cells and one of the sources of culture material for the realization, it is a primary culture in vitro, which can come from: tissues with high proliferative capacity, tissues with low activity (in this case ovaries and other organs of FRT), cells free from tissues or organs and finally blood cells or serous fluid.

The preparation of a primary culture takes place following precise steps. To isolate a homogeneous population from a tissue it is necessary to destroy the ECM and the junctions that hold the cells together, using different techniques: by migration from an explant, by mechanical disintegration and finally enzymatic disintegration. The cells able to survive the disintegration and proliferate will originate in a cell pool called primary culture («Primary Culture», 2010).

The possibility of physically isolating the populations of interest or the single cells taken is of great importance in the field of cell cultures and in the advent of 3D modeling such as organoids.

The strategies, in a general way, for cell separation from a mixed population are: exploit the different aptitude of different cell types to growth in vitro and separate cells on the basis of survival capacity in particular conditions such as the insertion of growth factors specifics reported in the works for organoid FRTs; exploit the difference in the different ability to adhere or not to growth substrates; use different enzymes for dissociation and always linked to this the use of specific growth media mentioned in the previous works.

After a careful analysis for the preparation, the fundamental steps for the preparation of the primary culture line are Sample collection, mechanical dissection, enzymatic treatment, enzyme inhibition, control of cell number and viability, seeding, evolution control over time, expansion, bio banking.

While several methods, such as the use of low adhesion round bottom plates and bioreactors, have been employed for the generation of three-dimensional models, an organoid can be grown in tissue culture plates embedded in the purified extracellular matrix in the form of a hydrogel and immersed in a specific culture medium containing the growth factors of the model to be studied (A. M. Capuzzo \& Vigo, 2020). The organoid dome technique can also be used with 
different models, providing communication between compartments and desired crosstalk, and submerged organoids can remain in the culture for the long term by adapting to the development and maturation timelines necessary for the formation of the desired system (The Organoid Culture Handbook, s.d.).

Organoids are frequently grown in matrix hydrogels, which encourage the formation of threedimensional structures but must be removed prior to passing, cryopreservation, and organoid analysis. Extracellular proteins can be degraded by proteases within the matrix organoid («ThreeDimensional Culture», 2010).

Organoids can be cryopreserved for cell line banking or the creation of repositories. For organoid cryopreservation, the same methods and chemicals that are used to freeze down cell lines and primary cells can be utilized, such as a base medium containing 20\% FBS and 10\% DMSO. Troubleshooting cell viability during cryopreservation is a technological issue due to their complicated structural components. Tissue type, organoid maturation, shape (freezing of entire structures, partially dissociated fragments, or totally dissociated single cell suspensions), and density may all need different freezing media and freeze-down strategies (5 Steps to Live-Cell Imaging, s.d.).

It's crucial to keep track of and manage organoid viability to establish consistent and reliable culture procedures. It's also necessary when employing 3D culture models for drug development or toxicity testing. The MTT Assay, which is used to identify metabolically active cells in undamaged and unfixed organoid tissue, is a common approach for determining cell viability. Using this colorimetric test, with tetrazolium salt, it allows to estimate the number of viable cells present within the structure, and this therefore allows to evaluate the efficacy of the current culture on the vitality of the population («Cytotoxicity», 2010).

For high-resolution imaging of immunostained organoids, confocal and light sheet microscopy are advised. Furthermore, preserving intact organoids for immunostaining to assess marker expression is a major issue in the realm of 3D structures. The use of robust and specific primary antibodies against tissue-specific markers will enhance tissue imaging and analysis, in addition to maintaining tissue integrity and guaranteeing matrix clarity for imaging («Equipment and Materials», 2010). 
Live cell imaging over longer periods of time than confocal imaging is one of the approaches that is currently gaining favor. This approach can be challenging since the microscope's objective might drift out of focus throughout the experiment, causing us to lose sight of our sample. This may be somewhat solved because the newest microscopes feature autofocus capabilities, making it simpler to maintain the lens in focus for longer periods of time and reducing focal drift (A. M. Capuzzo \& Vigo, 2021).

A time lapse recording technology has been extensively developed in recent years to depict natural processes and monitor them at a faster frame rate. He has changed the idea of observable events and promoted the method since the creation of bioluminescent proteins, which allow the fluorescent tagging of structures in the subcellular compartments of live cells (A. Capuzzo et al., 2020).

\section{Development of Co-Culture Crosstalk system between Organoids}

Intestinal organoids were recently co-cultured with stromal cells were created (Stzepourginski et al., 2017, pag. 34). Co-culture of organoids with stromal cells might add to the organoid models and provide a useful model for studying reciprocal epithelium-stroma interactions in vivo (Arnold et al., 2001).

Bioengineering techniques may make it possible to create more sophisticated tissue-like models including non-epithelial populations such fibroblasts, immunological cells, and endothelial cells. Co-culture of organoids may be used to study interactions between two distinct tissues, which is especially important for maternal-fetal crosstalk utilizing placental (trophoblast) and endometrial organoids (Alzamil et al., 2021).

The uterus is lined with the endometrium, a specialized tissue made up of a layer of epithelial cells and underlying stromal cells. During a menstrual cycle, the endometrium undergoes several changes to prepare for the implantation of an embryo and eventual pregnancy. Endometrium thickening and angiogenesis, or the formation of new blood vessels, are two of these changes. The blastocyst implants into the endometrium to prepare for the development of the placenta, which connects the mother's and fetal blood supply. The trophoblast, the blastocyst's outer layer, invades the endometrium and drives endometrial remodeling in preparation for implantation.Zambuto and colleagues set out to create a $3 \mathrm{D}$ model to investigate the endometrium and trophoblast crpsstalk, including endometrial angiogenesis and trophoblast 
invasion, in a recent research (Zambuto et al., 2019). They created a hydrogel platform that best matched endometrial rigidity using tissue engineering methods. After that, they co-cultured human umbilical vein endothelial cells (HUVECs) and endometrial stromal cells to validate the supports and promote the development of endothelial cell networks, which they used to mimic angiogenesis. They next assessed the model's development and dissemination, discovering that cells had connected to the matrix and had begun to spread out.

Endometrial stromal cells develop into decidual cells in response to progesterone and cAMP in preparation for pregnancy. Endometrial stromal cells were treated with progesterone and cAMP to see if their 3D platform might promote this stromal cell development. These therapies caused decidual cell morphology and elevated prolactin and IGFBP-1 levels, according to the researchers. Finally, they introduced trophoblast cells to their model to see if it could be used to investigate trophoblast invasion and found that cell invasion into the matrix increased (Alzamil et al., 2021).

The findings of Zambuto and colleagues, taken together, point to a novel model system for investigating endometrial dynamics and trophoblast invasion that combines two distinct models.

\section{Secretome Protein Expression}

Secretome-based screening was originally described in the post genomics era, when secreted proteins could be identified using bioinformatics, generated in high-throughput using recombinant expression, and examined in a variety of cell-based tests to see if they had an effect. Secreted proteins act as agonists or antagonists in the organism, initiating or suppressing physiological actions at the cellular level. As a result, cell-based assays, such as those utilized in a secretome-based method, are appropriate for measuring many types of responses (Mancini \& Pensabene, 2019).

Secreted proteins can also function as decoy factors in signaling pathways, leading in an antagonist reaction. Multiple decoy factors of the transforming growth factor (TGF-) superfamily control signaling via receptors by binding to cognate ligands and inhibiting a response. A similar effect is seen when the extracellular domains (ECDs) of single-pass plasma TM proteins are removed from the membrane. The results of a screen can discover proteins of therapeutic relevance at various points along the pipeline (Turco et al., 2018).

The primary screen's actives may be of interest. ECDs can be used in follow-up screening procedures to identify the cognate receptor. Transcriptome profiling can identify genes in 
pathways that are elevated by secretome proteins, which can then be verified using siRNA and/or cell line models lacking the gene of interest. In the literature, affinities and/or Kd values between ligand cognate receptor pairs range from low picomolar to low micromolar. Before doing an unbiased secretome screen, it is important to quantify the concentration of each sample in the secreted protein library. This eliminates false negatives caused by low protein concentrations in the assay. Therefore, most efforts have developed methods for determining the amount of protein generated (Deloria et al., 2021).

The Haptoglobin protein on some occasions, in the oviduct and in the uterus of pigs, has been evaluated on its possible impact and role in the in vitro embryogenesis of pigs. In several studies, an orderly ratio of sows in the various estrous stages was made late follicular stage, early luteum and late luteum. The haptoglobin mRNA was then tested using RT-qPCR. In addition, immunohistochemistry was used to break down the protein joint, and the results were complemented by Western-Blot and proteomic analyses of oviductal and uterine secretions. The findings show that haptoglobin is plentiful in the porcine oviduct and uterus during the late luteal phase of the estrus cycle. Despite the comparator and reference groups, haptoglobin expansion during gamete co-hatching had no effect on sperm infiltration, monospermia, or efficacy rates; blastocyst advancement was basically further developed when haptoglobin was available (haptoglobin: 64.50 percent vs. control: 37.83 percent ; p0.05). Overall, the presence of haptoglobin in the oviduct and uterus of sows at different phases of the estrus cycle shows that it is important in the propagation interaction (Bazer \& Johnson, 2014).

LIF mRNA is distinguished in the human endometrium, expanding during the secretory phase (Arici et al., 1995). Even more explicitly, LIF and LIFR communicate at most during the "implantation window" and are confronted with the presence of the blastocyst (Aghajanova, 2004). Immunohistochemical examination of LIF in 89 biopsies of mature women reveals its most notable articulation during the middle luteal stage in the luminal epithelium, fundamentally more prominent than the organs or stroma (Arici et al., 1995). In this way, LIF is positioned to directly mark the peri-implant blastocyst. The secretion of LIF from the glandular endometrium depends on hormone levels via paracrine signals and on progesterone, as treatment with mifepristone, an enemy of the progesterone receptor, reduces the emission of LIF from the glandular endometrium (Arici et al., 1995). Endometrial LIF is susceptible to several developmental variables and molecular cytokine signals, including interleukin-1, tumor necrosis 
factor (TNF), platelet-determined development factor, and epidermal development factor (EGF), while it is hindered by interferon gamma (Karpuzoglu-Sahin et al., 2001).

\section{Improved Fertilization In Vitro}

Women with spontaneous or premature ovarian failure had no effective reproductive treatments until 1983, despite early IVF findings improving the technology for treating tubal disease. In December of that year, a 25-year-old woman with secondary amenorrhea and premature ovarian failure became the first person to successfully deliver a pregnancy using a donor egg. Dr. Peter Renou of the Monash IVF group in Australia successfully inseminated a single egg donated by a 29-year-old lady who was undergoing IVF for tubal disease with the recipient's husband's sperm. The embryo was placed into the uterus of the recipient, resulting in a live full-term kid. (Karaki et al., 2002).

Over the last two decades, the major indication for oocyte donation has shifted from women with early ovarian failure to women of beyond reproductive age. One of the elements driving this tendency is the changing demographics of the population. More women are deferring children to pursue education and employment; marriages are occurring later in life; divorce and remarriage are becoming more prevalent; and modern contraception and abortion services have eliminated many unintended pregnancies. Traditional IVF is still a possibility for older individuals, however pregnancy chances drop substantially beyond 36 years of age due to the normal oocytes' age-related decline (Wang \& Sauer, 2006).

Pregnancy rates among women utilizing donor oocytes, on the other hand, have been reported to be as high as $50 \%$ per embryo transfer across all age groups. Women in their sixties have given birth after receiving donor oocytes, demonstrating that the postmenopausal uterus may still support pregnancy with the appropriate hormonal support. Oocyte recipients, on the other hand, have a greater risk of obstetrical complications such as cesarean section (40-76\%), pregnancyinduced hypertension (16-40\%), and gestational diabetes (40-76\%)(Karaki et al., 2002).

For the assessment of endometrial receptivity, early research using transvaginal ultrasonography (TVUS) measurement of endometrial thickness aimed to replace invasive procedures like endometrial biopsy. A thickness of $7 \mathrm{~mm}$ in the pre-ovulatory endometrium is considered the cut-off below which poor implantation occurs. Patients with endometrial thickness as low as 4 mm 60 have been observed to become pregnant. In 2014, a meta-analysis found that endometrial 
thickness did not have a high enough positive predictive value for conception on its own (Kyrou et al., 2009).

More recent research has focused on TVUS measurements of sub endometrial contractility, which manifests as "endometrial waves" with hormonally responsive patterns and propagation directions. These peristaltic waves are directed from the fundus to the cervix (FC waves) during the follicular phase, but from the cervix to the fundus (CF waves) during the late follicular and ovulatory phases to aid sperm transit to the fallopian tube. Following ovulation, the uterus enters a state of quiescence that is conducive to embryo implantation (Kyrou et al., 2009).

The clinical and laboratory methodology for ART continued to advance, and a surplus of embryos more than what is used or required for the initial IVF therapy grew more common. During the early days of IVF, patients with extra embryos had the option of rejecting them, donating them to another infertile couple, or donating them for scientific purposes. Although embryo cryopreservation was an option, the freezing and thawing methods generally resulted in lasting cell damage, and most embryos did not survive. This is best demonstrated by the extremely low pregnancy rates observed following the transfer of frozen/thawed embryos in the 1980s (Eskew \& Jungheim, 2017).

Intensive efforts to create various freezing/thawing procedures and cryoprotective substances finally resulted in the first human pregnancy from a frozen embryo in 1983, which unfortunately ended in premature membrane rupture and pregnancy termination at 24 weeks of gestation. Despite the setback, cryopreservation technology advanced in the 1980s, leading to an increase in embryo survival and conception rates (Eskew \& Jungheim, 2017).

Only approximately half of the embryos survived the freeze/thaw process in the early years of research, resulting in a pregnancy rate of $13.4 \%$ per embryo transfer operation, with only 4.6 percent of the individual thawed embryos implanting. In the United States in 2003, frozen embryo transfers accounted for 21981 of the 112872 IVF cycles $(17.8 \%)$, with a 27.0 percent overall live birth rate per embryo transfer procedure (Xiao et al., 2017). 


\section{Conclusions}

Despite current restrictions, organoids give an interesting time as in vitro models of the conceptive framework that consider conceptive science research, exact displaying of regenerative organs, clinical dynamic, and individualized medication for patients with gynecologic malignant growths furthermore, infections like endometriosis. Likewise, bio-banked organoids can be utilized for drug screening or in vitro preliminaries to foresee singular patient medication reactions. Advances on state-of-the-art advances will without a doubt improve this original instrument for clinical application.

The FRT varies dramatically during life, and it's appropriate functioning is critical for reproductive health and happiness. The absence of physiologically realistic models, as well as reasonable ethical concerns, have impeded research in this subject. The development of FRT organoids that can represent both healthy and sick tissues will be revolutionary. Organoids are a strong model system because they may recapitulate important morphological, molecular, and functional aspects of tissues while also being adaptable to a wide range of downstream applications.

Organoids are more expensive and time-consuming than 2D cell lines, but the method is simple and can be replicated by other laboratories. We will be able to investigate the fundamental biology of how these tissues function, as well as how various cell types interact, and get insight into cancer and infectious disease etiology, using FRT organoids. They also present prospects for translational research in medication discovery and personalized medicine. These new technologies open new avenues for addressing unresolved concerns in the FRT's study field, holding enormous promise for enhancing female's health and well-being.

Without 3D cell culture technologies, in vitro modeling of critical developmental processes as well as clinical disease has become difficult. Because of the accurate simulation of a live tissue's complex environment, 3D cell cultures have accelerated biology research and led to more advanced procedures. Incorporating all of the different cell types found in a complex tissue, realistic modeling of regional differences in ECM composition, stiffness, and organization, and constructing robust vascular networks to ensure nutrient delivery throughout the construct are all challenges that remain in developing fully physiologically relevant 3D cell cultures systems. 
The disclosed and discussed in vitro 3D FRT cell culture might serve as a springboard for the creation of novel methods and experimental designs tailored to specific demands.

\section{Bibliography}

3D Model Systems: Spheroids, Organoid and Tissue Model Systems. (2020, giugno 2). Cell Culture Dish. https://cellculturedish.com/3d-model-systems-spheroids-organoid-and-tissue-modelsystems/

5 steps to live-cell imaging. (s.d.). 12.

Abbas, Y., Turco, M. Y., Burton, G. J., \& Moffett, A. (2020). Investigation of human trophoblast invasion in vitro. Human Reproduction Update, 26(4), 501-513. https://doi.org/10.1093/humupd/dmaa017

Aghajanova, L. (2004). Leukemia inhibitory factor and human embryo implantation. Annals of the New York Academy of Sciences, 1034, 176-183. https:/ / doi.org/10.1196/annals.1335.020

Alzamil, L., Nikolakopoulou, K., \& Turco, M. Y. (2021). Organoid systems to study the human female reproductive tract and pregnancy. Cell Death $\mathcal{E}$ Differentiation, 28(1), 35-51. https://doi.org/10.1038/s41418-020-0565-5

Arici, A., Engin, O., Attar, E., \& Olive, D. L. (1995). Modulation of leukemia inhibitory factor gene expression and protein biosynthesis in human endometrium. The Journal of Clinical Endocrinology and Metabolism, 80(6), 1908-1915. https://doi.org/10.1210/jcem.80.6.7775640

Arnold, J. T., Kaufman, D. G., Seppälä, M., \& Lessey, B. A. (2001). Endometrial stromal cells regulate epithelial cell growth in vitro: A new co-culture model. Human Reproduction (Oxford, England), 16(5), 836-845. https:// doi.org/10.1093/humrep/16.5.836

Bazer, F. W., \& Johnson, G. A. (2014). Pig blastocyst-uterine interactions. Differentiation; Research in Biological Diversity, 87(1-2), 52-65. https:/ / doi.org/10.1016/j.diff.2013.11.005

Burkitt, M., Walker, D., Romano, D. M., \& Fazeli, A. (2012). Using computational modeling to investigate sperm navigation and behavior in the female reproductive tract. Theriogenology, 77(4), 703-716. https:/ / doi.org/10.1016/j.theriogenology.2011.11.011

Capuzzo, A. M., \& Vigo, D. (2020). Updated modifications about Spinœ Bioreactor. Journal of Engineering Technology and Applied Sciences, 5, 53-56.

Capuzzo, A. M., \& Vigo, D. (2021). Microfluidic Live-Imaging Technology to Perform Research Activities in 3D Models [Preprint]. other. https://doi.org/10.20944/preprints202103.0264.v2

Capuzzo, A. M., Vigo, D., Brecchia, G., Curone, G., \& Pasqua, S. (2020). Microfluidic live-imaging with CELLviewer technology to perform biotechnological tasks. Journal of Engineering Technology and Applied Sciences, 5, 27-34. 
Capuzzo, A., Vigo, D., \& Curone, G. (2020). Automation in 3D cellular system in Live-Imaging with Microfluidic Technology CELLviewer ${ }^{\circledR}$. https://doi.org/10.13140/RG.2.2.21516.54408

Chen, S., \& Schoen, J. (2019). Air-liquid interface cell culture: From airway epithelium to the female reproductive tract. Reproduction in Domestic Animals = Zuchthygiene, 54 Suppl 3, 38-45. https://doi.org/10.1111/rda.13481

Choi, J., Iich, E., \& Lee, J. H. (2016). Organogenesis of adult lung in a dish: Differentiation, disease and therapy. Developmental Biology, 420(2), 278-286. Scopus. https:// doi.org/10.1016/j.ydbio.2016.10.002

Chumduri, C., \& Turco, M. (2021). Organoids of the female reproductive tract. Journal of Molecular Medicine, 99, 1-23. https:/ / doi.org/10.1007/s00109-020-02028-0

Cytotoxicity. (2010). In Culture of Animal Cells (pagg. 365-381). John Wiley \& Sons, Ltd. https://doi.org/10.1002/9780470649367.ch21

Deloria, A. J., Haider, S., Dietrich, B., Kunihs, V., Oberhofer, S., Knofler, M., Leitgeb, R., Liu, M., Drexler, W., \& Haindl, R. (2021). Ultra-High-Resolution 3D Optical Coherence Tomography Reveals Inner Structures of Human Placenta-Derived Trophoblast Organoids. IEEE Transactions on Bio-Medical Engineering, 68(8), 2368-2376. https:/ / doi.org/10.1109/TBME.2020.3038466

Desai, N., Abdelhafez, F., Calabro, A., \& Falcone, T. (2012). Three dimensional culture of fresh and vitrified mouse pre-antral follicles in a hyaluronan-based hydrogel: A preliminary investigation of a novel biomaterial for in vitro follicle maturation. Reproductive Biology and Endocrinology : RBEE, 10, 29. https://doi.org/10.1186/1477-7827-10-29

Desai, S. P., Bhatia, S. N., Toner, M., \& Irimia, D. (2013). Mitochondrial localization and the persistent migration of epithelial cancer cells. Biophysical Journal, 104(9), 2077-2088. Scopus. https://doi.org/10.1016/j.bpj.2013.03.025

Elad, D., Jaffa, A. J., \& Grisaru, D. (2020). Biomechanics of Early Life in the Female Reproductive Tract. Physiology (Bethesda, Md.), 35(2), 134-143. https:// doi.org/10.1152/ physiol.00028.2019

Equipment and Materials. (2010). In Culture of Animal Cells (pagg. 37-56). John Wiley \& Sons, Ltd. https:/ / doi.org/10.1002/9780470649367.ch4

Eskew, A. M., \& Jungheim, E. S. (2017). A History of Developments to Improve in vitro Fertilization. Missouri Medicine, 114(3), 156-159.

Fritz, R., Jain, C., \& Armant, D. R. (2014). Cell signaling in trophoblast-uterine communication. The International Journal of Developmental Biology, 58(2-3-4), 261-271. https://doi.org/10.1387/ijdb.140011da

Haider, S., Meinhardt, G., Saleh, L., Kunihs, V., Gamperl, M., Kaindl, U., Ellinger, A., Burkard, T. R., Fiala, C., Pollheimer, J., Mendjan, S., Latos, P. A., \& Knöfler, M. (2018). Self-Renewing Trophoblast Organoids Recapitulate the Developmental Program of the Early Human Placenta. Stem Cell Reports, 11(2), 537-551. https:/ / doi.org/10.1016/j.stemcr.2018.07.004 
Haycock, J. W. (2011). 3D Cell Culture: A Review of Current Approaches and Techniques. In J. W. Haycock (A c. Di), 3D Cell Culture: Methods and Protocols (pagg. 1-15). Humana Press. https://doi.org/10.1007/978-1-60761-984-0_1

Heidari-Khoei, H., Esfandiari, F., Hajari, M. A., Ghorbaninejad, Z., Piryaei, A., \& Baharvand, H. (2020). Organoid technology in female reproductive biomedicine. Reproductive Biology and Endocrinology, 18(1), 64. https://doi.org/10.1186/s12958-020-00621-z

Heremans, R., Jan, Z., Timmerman, D., \& Vankelecom, H. (2021). Organoids of the Female Reproductive Tract: Innovative Tools to Study Desired to Unwelcome Processes. Frontiers in Cell and Developmental Biology, 9, 867. https://doi.org/10.3389/fcell.2021.661472

Herrler, A., von Rango, U., \& Beier, H. M. (2003). Embryo-maternal signalling: How the embryo starts talking to its mother to accomplish implantation. Reproductive BioMedicine Online, 6(2), 244256. https:/ / doi.org/10.1016/S1472-6483(10)61717-8

Horii, M., Touma, O., Bui, T., \& Parast, M. M. (2020). Modeling human trophoblast, the placental epithelium at the maternal fetal interface. Reproduction, 160(1), R1-R11. https://doi.org/10.1530/REP-19-0428

Karaki, R. Z., Samarraie, S. S., Younis, N. A., Lahloub, T. M., \& Ibrahim, M. H. (2002). Blastocyst culture and transfer: A step toward improved in vitro fertilization outcome. Fertility and Sterility, 77(1), 114-118. https:/ / doi.org/10.1016/s0015-0282(01)02939-9

Karpuzoglu-Sahin, E., Hissong, B. D., \& Ansar Ahmed, S. (2001). Interferon-gamma levels are upregulated by 17-beta-estradiol and diethylstilbestrol. Journal of Reproductive Immunology, 52(12), 113-127. https:/ / doi.org/10.1016/s0165-0378(01)00117-6

Kessler, M., Hoffmann, K., Brinkmann, V., Thieck, O., Jackisch, S., Toelle, B., Berger, H., Mollenkopf, H.-J., Mangler, M., Sehouli, J., Fotopoulou, C., \& Meyer, T. F. (2015). The Notch and Wnt pathways regulate stemness and differentiation in human fallopian tube organoids. Nature Communications, 6(1), 8989. https://doi.org/10.1038/ncomms9989

Kwong, J., Chan, F. L., Wong, K., Birrer, M. J., Archibald, K. M., Balkwill, F. R., Berkowitz, R. S., \& Mok, S. C. (2009). Inflammatory cytokine tumor necrosis factor alpha confers precancerous phenotype in an organoid model of normal human ovarian surface epithelial cells. Neoplasia (New York, N.Y.), 11(6), 529-541. https:/ / doi.org/10.1593/neo.09112

Kyrou, D., Kolibianakis, E. M., Venetis, C. A., Papanikolaou, E. G., Bontis, J., \& Tarlatzis, B. C. (2009). How to improve the probability of pregnancy in poor responders undergoing in vitro fertilization: A systematic review and meta-analysis. Fertility and Sterility, 91(3), 749-766. https:// doi.org/10.1016/j.fertnstert.2007.12.077

Lancaster, M., \& Knoblich, J. (2014). Organogenesis in a dish: Modeling development and disease using organoid technologies. Science. https://doi.org/10.1126/science.1247125

Łaniewski, P., Gomez, A., Hire, G., So, M., \& Herbst-Kralovetz, M. M. (2017). Human ThreeDimensional Endometrial Epithelial Cell Model To Study Host Interactions with Vaginal Bacteria 
and Neisseria gonorrhoeae. Infection and Immunity, 85(3), e01049-16. https://doi.org/10.1128/IAI.01049-16

Laronda, M. M., Burdette, J. E., Kim, J., \& Woodruff, T. K. (2013). Recreating the female reproductive tract in vitro using iPSC technology in a linked microfluidics environment. Stem Cell Research \& Therapy, 4 Suppl 1, S13. https:// doi.org/10.1186/scrt374

Li, Q., Agno, J. E., Edson, M. A., Nagaraja, A. K., Nagashima, T., \& Matzuk, M. M. (2011). Transforming Growth Factor $\beta$ Receptor Type 1 Is Essential for Female Reproductive Tract Integrity and Function. PLOS Genetics, 7(10), e1002320. https://doi.org/10.1371/journal.pgen.1002320

Madan, P. (2011). Arrest or Survive: A Decision of the Early Preimplantation Embryo That Influences Fertility. In Comprehensive Biotechnology (pagg. 481-488). Elsevier. https://doi.org/10.1016/B978-0-444-64046-8.00236-6

Madsen, C. A., Perry, G. A., Mogck, C. L., Daly, R. F., MacNeil, M. D., \& Geary, T. W. (2015). Effects of preovulatory estradiol on embryo survival and pregnancy establishment in beef cows. Animal Reproduction Science, 158, 96-103. https://doi.org/10.1016/j.anireprosci.2015.05.006

Maenhoudt, N., Defraye, C., Boretto, M., Jan, Z., Heremans, R., Boeckx, B., Hermans, F., Arijs, I., Cox, B., Van Nieuwenhuysen, E., Vergote, I., Van Rompuy, A.-S., Lambrechts, D., Timmerman, D., \& Vankelecom, H. (2020). Developing Organoids from Ovarian Cancer as Experimental and Preclinical Models. Stem Cell Reports, 14(4), 717-729. https://doi.org/10.1016/j.stemcr.2020.03.004

Mancini, V., \& Pensabene, V. (2019). Organs-On-Chip Models of the Female Reproductive $\begin{array}{lllll}\text { System. Bioengineering } & \text { (Basel, } & \text { Switzerland), } & 6(4), & \text { E103. }\end{array}$ https:// doi.org/10.3390/bioengineering6040103

Meyer, A. E., Pfeiffer, C. A., Brooks, K. E., Spate, L. D., Benne, J. A., Cecil, R., Samuel, M. S., Murphy, C. N., Behura, S., McLean, M. K., Ciernia, L. A., Smith, M. F., Whitworth, K. M., Wells, K. D., Spencer, T. E., Prather, R. S., \& Geisert, R. D. (2019). New perspective on conceptus estrogens in maternal recognition and pregnancy establishment in the pig†. Biology of Reproduction, 101(1), 148-161. https://doi.org/10.1093/biolre/ioz058

Primary Culture. (2010). In Culture of Animal Cells (pagg. 163-186). John Wiley \& Sons, Ltd. https://doi.org/10.1002/9780470649367.ch11

Schomberg, D. W. (1978). Basic mechanisms of ovarian endocrine function. Environmental Health Perspectives, 24, 5-10. https:// doi.org/10.1289/ehp.78245

Senol, S., Sayar, I., Ceyran, A. B., Ibiloglu, I., Akalin, I., Firat, U., Kosemetin, D., Engin Zerk, P., \& Aydin, A. (2016). Stromal Clues in Endometrial Carcinoma: Loss of Expression of $\beta$-Catenin, Epithelial-Mesenchymal Transition Regulators, and Estrogen-Progesterone Receptor. International Journal of Gynecological Pathology, 35(3), 238-248. https:// doi.org/10.1097/PGP.0000000000000233 
Sheridan, M. A., Fernando, R. C., Gardner, L., Hollinshead, M. S., Burton, G. J., Moffett, A., \& Turco, M. Y. (2020). Establishment and differentiation of long-term trophoblast organoid cultures from the human placenta. Nature Protocols, 15(10), 3441-3463. https://doi.org/10.1038/s41596020-0381-x

Stejskalová, A., Vankelecom, H., Sourouni, M., Ho, M. Y., Götte, M., \& Almquist, B. D. (2021). In vitro modelling of the physiological and diseased female reproductive system. Acta Biomaterialia, S1742-7061(21)00269-5. https:/ / doi.org/10.1016/j.actbio.2021.04.032

Stzepourginski, I., Nigro, G., Jacob, J.-M., Dulauroy, S., Sansonetti, P. J., Eberl, G., \& Peduto, L. (2017). CD34+ mesenchymal cells are a major component of the intestinal stem cells niche at homeostasis and after injury. Proceedings of the National Academy of Sciences of the United States of America, 114(4), E506-E513. https://doi.org/10.1073/pnas.1620059114

The Organoid Culture Handbook. (s.d.). Www.Rndsystems.Com. Recuperato 14 gennaio 2021, da https://www.rndsystems.com/resources/literature/organoid-culture-handbook

Three-Dimensional Culture. (2010). In Culture of Animal Cells (pagg. 481-495). John Wiley \& Sons, Ltd. https:// doi.org/10.1002/9780470649367.ch25

Turco, M. Y., Gardner, L., Kay, R. G., Hamilton, R. S., Prater, M., Hollinshead, M. S., McWhinnie, A., Esposito, L., Fernando, R., Skelton, H., Reimann, F., Gribble, F. M., Sharkey, A., Marsh, S. G. E., O'Rahilly, S., Hemberger, M., Burton, G. J., \& Moffett, A. (2018). Trophoblast organoids as a model for maternal-fetal interactions during human placentation. Nature, 564(7735), 263-267. https:/ / doi.org/10.1038/s41586-018-0753-3

Wang, J., \& Sauer, M. V. (2006). In vitro fertilization (IVF): A review of 3 decades of clinical innovation and technological advancement. Therapeutics and Clinical Risk Management, 2(4), 355364. https://doi.org/10.2147/tcrm.2006.2.4.355

Wong, A. S., \& Auersperg, N. (2003). Ovarian surface epithelium: Family history and early events in ovarian cancer. Reproductive Biology and Endocrinology, 1(1), 70. https:/ / doi.org/10.1186/14777827-1-70

Xiao, S., Coppeta, J. R., Rogers, H. B., Isenberg, B. C., Zhu, J., Olalekan, S. A., McKinnon, K. E., Dokic, D., Rashedi, A. S., Haisenleder, D. J., Malpani, S. S., Arnold-Murray, C. A., Chen, K., Jiang, M., Bai, L., Nguyen, C. T., Zhang, J., Laronda, M. M., Hope, T. J., ... Woodruff, T. K. (2017). A microfluidic culture model of the human reproductive tract and 28-day menstrual cycle. Nature Communications, 8, 14584. https://doi.org/10.1038/ncomms14584

Yin, X., Mead, B. E., Safaee, H., Langer, R., Karp, J. M., \& Levy, O. (2016). Engineering Stem Cell Organoids. Cell Stem Cell, 18(1), 25-38. https:/ / doi.org/10.1016/j.stem.2015.12.005

Zambuto, S. G., Clancy, K. B. H., \& Harley, B. A. C. (2019). A Gelatin Hydrogel to Study Endometrial Angiogenesis and Trophoblast Invasion (pag. 548024). https:/ / doi.org/10.1101/548024 